\title{
Twirling, whirling, and tensioning: Plectoneme formation and suppression in flexible filaments
}

\author{
Isaac R. Bruss, ${ }^{1, *}$ Heena K. Mutha, ${ }^{2, *}$ Katherine Stoll, ${ }^{2}$ Brent Collins, ${ }^{2}$ Vinh Nguyen $\odot,{ }^{2}$ David J. D. Carter $\odot,{ }^{2}$ \\ Michael P. Brenner, ${ }^{1,3}$ and Kasey J. Russell ${ }^{2, \dagger}$ \\ ${ }^{1}$ School of Engineering and Applied Sciences, Harvard University, Cambridge, Massachusetts 02138, USA \\ ${ }^{2}$ The Charles Stark Draper Laboratory, Incorporated, Cambridge, Massachusetts 02139, USA \\ ${ }^{3}$ Kavli Institute of Bionano Sciences and Technology, Harvard University, Cambridge, Massachusetts 02138, USA
}

(Received 11 July 2019; published 19 November 2019)

\begin{abstract}
A high-aspect ratio flexible filament suspended in a viscous fluid is unstable to a symmetry-breaking whirling motion when axially rotated beyond a critical velocity. We present experiments of this behavior and uncover a rich diversity of plectoneme formation soon after the initial instability, including multiple plectonemes for especially long filaments. A physical model extending Timoshenko's theory of elastic instability is presented which captures the fundamental physics involved in the instability. Additionally, we consider the effects of an axial flow that acts to tension the filament and control the onset of whirling without adjusting the physical properties of the filament itself. Such a setup could be employed to selectively engineer plectoneme formation at specified locations and lengths, and in fibers with submicron diameters, potentially to create superelastic ropes and fabrics.
\end{abstract}

DOI: 10.1103/PhysRevResearch.1.032020

A sufficiently flexible object immersed in a moving fluid will dynamically change shape-and therefore alter the encompassing flow field-despite the standard assumption that low Reynolds number fluids are characterized as having a smooth and steady flow field [1]. A common example is the motion of microscopic cilia and flagella, which are able to transform their rotational and/or cyclic motion into translational motion of the entire cell [2].

A prevalent class of shape change, especially for largeaspect ratio elastic materials - as in the two biological examples above-is the buckling instability, which comes in two varieties: compressional Euler buckling, and helical buckling $[3,4]$. The dynamic and versatile behavior of Euler and helical buckling has been cleverly exploited by a variety of biological phenomena such as filopodia to produce motion [5], uniflagellated bacteria to achieve reorientation [6], and plant roots to redirect growth around barriers [7]. However, in general, torsion-induced helical buckling has historically been classified as a mode of "failure," for instance, in industrial applications such as breaking tubes during well drilling [8,9]. Similarly, high-aspect ratio wires formed from electrospun nanofibers of submicron diameter and centimeter-scale lengths coated with metal [10] can undergo torsion-induced failure when being twisted to synthesize micron-scale litz wire [11].

\footnotetext{
*These authors contributed equally to this work.

Corresponding author: krussell@ draper.com
}

Published by the American Physical Society under the terms of the Creative Commons Attribution 4.0 International license. Further distribution of this work must maintain attribution to the author(s) and the published article's title, journal citation, and DOI.
Both Euler and helical buckling modes can be produced via fluid flow. For example, Euler buckling has been observed experimentally by extruding a filament [12] or by rotating helical filaments [13] in a highly viscous fluid. In this Rapid Communication, we present experiments demonstrating fluidinduced helical buckling in straight filaments.

To study fluid-induced helical buckling at a laboratory scale, we cast straight filaments of silicone, a low Young's modulus material, with submillimeter diameters and centimeter-length scales. These high-aspect ratio filaments (length over radius $L / a>100$ ), were immersed and rotated in a recirculating tank filled with a high viscosity fluid, glycerin. The fluid and filament densities were similar, roughly within $6 \%$, in order to minimize buoyancy effects. A schematic of the system is given in Fig. 1(a), and the details are in the Supplemental Material (SM) [14]. By varying angular velocities and filament aspect ratios, we observed the presence of three different dynamical regimes: twirling, where the rod rotated around its center line as a speedometer cable [Fig. 1(b)]; whirling, where the rod writhed around the rotation axis as a crankshaft, which then evolved to overwhirling, where it then formed a steady state whirling "U" shape [Fig. 1(c)]; and finally plectonemes, where filaments self-contacted and wrapped around themselves similar to the twisted kinks in garden hoses [Fig. 1(d)]. For rotating filaments with a fixed geometry (length $L$ and radius $a$ ) and Young's modulus $E$, we typically observed the transition from stable twirling to unstable overwhirling and plectonemes with increasing angular velocity $\omega$ (Fig. 2). We were able to tune the critical angular velocity $\omega_{c}$ by immersing the filament in an axial fluid flow field that generated tension along the length of the filament.

Several insightful models have investigated the underlying physics and conjectured resulting modes of filaments under fluid-induced helical buckling. Most notably, Wolgemuth et al. [15] introduced the twirling and whirling modes in 

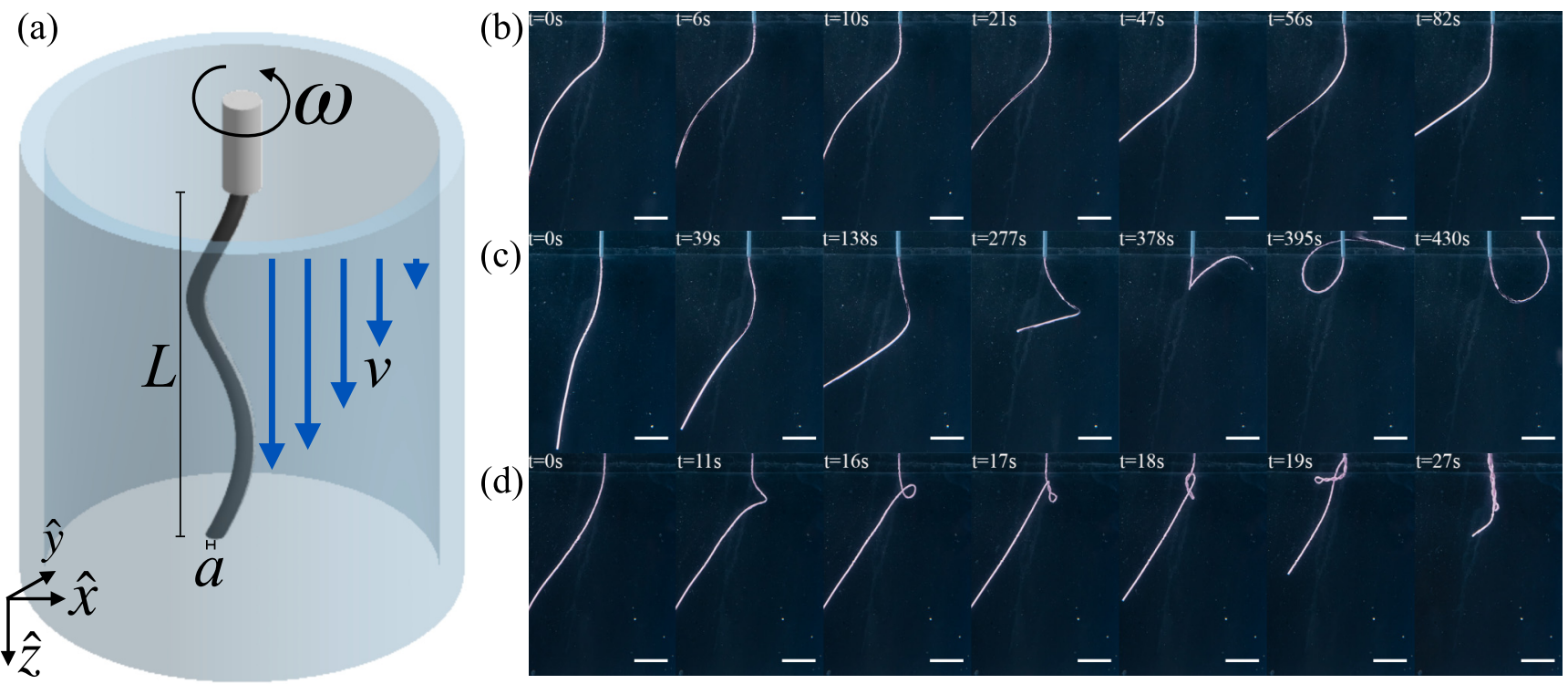

FIG. 1. (a) Diagram of the experimental setup. A flexible filament of length $l$ and radius $a$ is freely suspended in a viscous fluid and rotated at the top with an angular velocity $\omega$. A pump provides downward flow with a velocity $v$ to tension the filament. (b)-(d) Times series of 380- $\mu$ m-diam filaments with $v=0$, demonstrating (b) stable twirling motion with $L=8 \mathrm{~cm}$ and $\omega=12 \mathrm{rad} / \mathrm{s}$, (c) unstable whirling leading to overwhirling motion with $L=8 \mathrm{~cm}$ and $\omega=26 \mathrm{rad} / \mathrm{s}$, and (d) plectoneme formation with $L=11 \mathrm{~cm}$ and $\omega=61 \mathrm{rad} / \mathrm{s}$. Scale bars are all $2 \mathrm{~cm}$.

rotating filaments using numerical and analytical methods. Building upon this work, there have been multiple attempts to simulate the twirling to whirling instability [16], including a hydrodynamic bead-spring model [17,18], immersedboundary fluid dynamics [19], and a combination Kirchhoff rod/regularized stokeslet model [20]. These works all predicted the existence of the additional stable state, overwhirling. Finally, plectonemes, the far-from instability structures commonly found in DNA [21,22], are the anticipated final form of helically buckled rods that are torqued well beyond the initial buckling event $[4,23,24]$. Our experiments confirmed the existence of these predicted whirling, overwhirling, and plectoneme modes, and provide insight into the range of possible unstable modes. In addition, our experiments added tension to the filament with axial fluid flow. Here, using a simplified Kirchhoff rod and slender-body fluid dynamics model, we extend existing modeling efforts to explain the critical angular velocity for buckling $\omega_{c}$ as well as its dependence on the filament's aspect ratio, material properties, and fluid parameters.

We begin with a scaling analysis to establish the essential physical phenomena. We can estimate $\omega_{c}$ by building upon the classic theory of elastic instability for a flexible rod with hinged boundary conditions. Based on the standard Kirchhoff equations, a rod (see Fig. 1) of length $L$ and radius $a$ is in equilibrium when

$$
E I \frac{d^{2} x}{d z^{2}}=T x+C \tau \frac{d y}{d z}, \quad E I \frac{d^{2} y}{d z^{2}}=T y-C \tau \frac{d x}{d z},
$$

where $E$ is the Young's modulus, $I=\frac{\pi}{4} a^{4}$ is the second moment of area, $T$ is the axial tension, $C=E I /(1+v)$ is the twist modulus, $v$ is Poisson's ratio, and $\tau$ is the Frenet-Serret torsion of the curve [4]. Following the theory laid out by
Timoshenko [3], we can derive the magnitude of the critical torsion of the curve for helical buckling of a uniformly twisted rod that is freely suspended at both ends,

$$
\tau_{c}(L, T)=\frac{3 \sqrt{T L^{2}+E I \pi^{2}}}{L \sqrt{E I}}=\sqrt{\frac{36 T}{\pi a^{4} E}+\frac{9 \pi^{2}}{L^{2}}},
$$

where we have henceforth assumed an incompressible material with a Poisson's ratio of $v=0.5$, such as the effectively incompressible silicone rubber used in our experiments. In the limit of zero tension, Eq. (2) yields $\tau_{c}(L, 0)=3 \pi / L$, anticipating that for our finite length system there is always a critical nonzero torsion of the curve required to achieve buckling. Furthermore, we will show that Eq. (2), despite being derived for the case of a uniformly twisted rod, can serve as the foundation for an approximate solution to a more general system with nonuniform torsion.

Specifically, in our system torsion is generated by rotating the rod at the top $(s=0)$ through a fluid with a viscosity $\eta$. Assuming zero Reynolds number, slender-body dynamics gives the drag coefficient for an axially rotating rod at equilibrium to be $\zeta_{r}=4 \pi \eta a^{2}[25]$. Because the bottom $(s=L)$ of the rod is free, at equilibrium the magnitude of the internal torsion of the curve resulting from this rotational drag decreases linearly with $s$,

$$
\tau(s)=\frac{\zeta_{r} \omega}{C}(L-s)=24 \frac{\omega \eta}{E a^{2}}(L-s)
$$

Now we can ask, given a partial section of the rod between $s=0$ and some arc length $s=s^{*}$, and assuming it obeys Eq. (1), is the torsion strong enough to induce helical buckling? To utilize Eq. (2), we approximate the $s$-dependent torsion of the curve as being uniform within this $s^{*}$ segment 

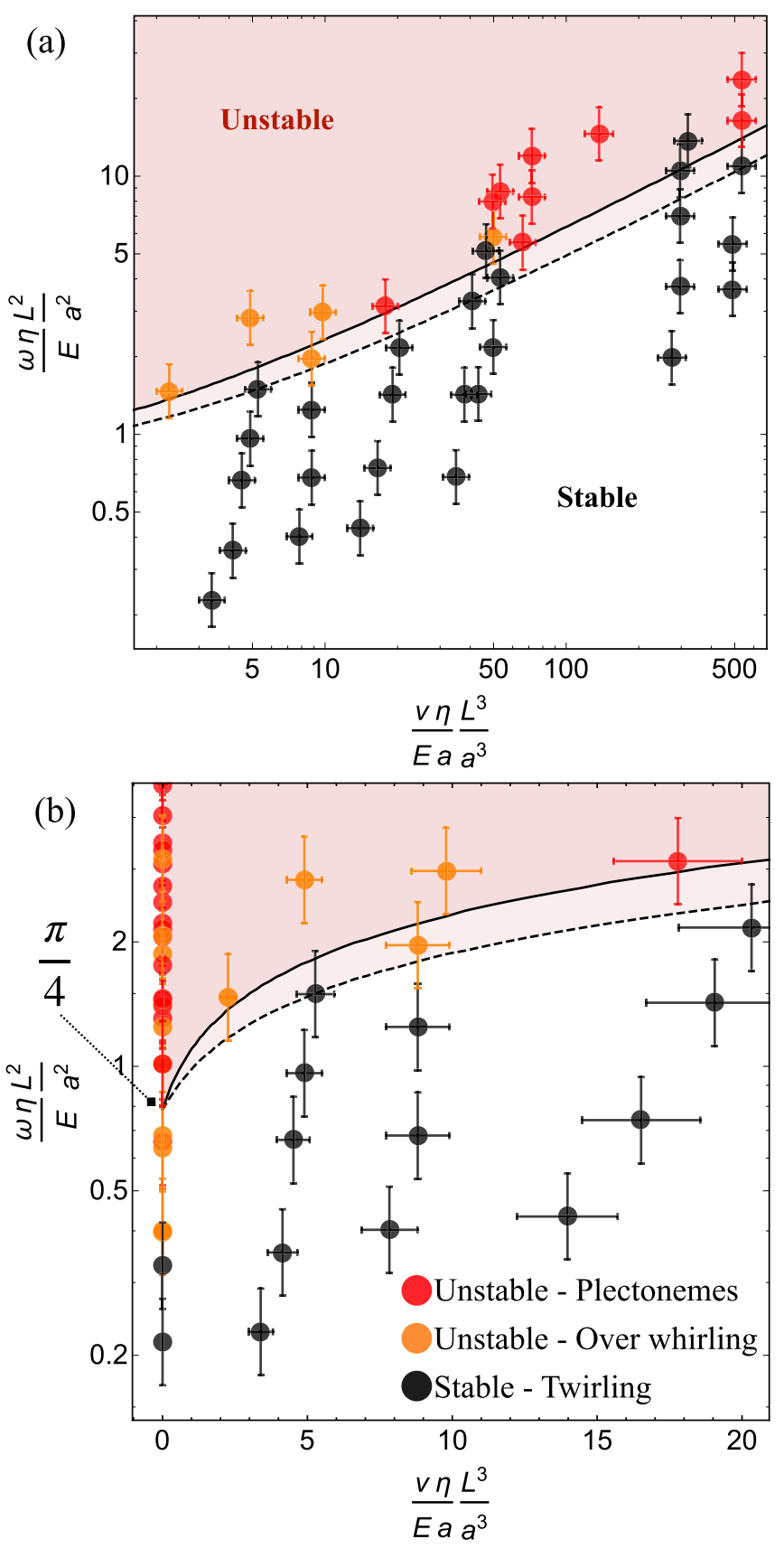

FIG. 2. Phase diagrams of instability with (a) $\log -\log$ and (b) $\log$ linear scaling. The solid and dashed lines show Eq. (7) with $\alpha=8$ for $(L / a)=100$ and $(L / a)=1000$, respectively. The region above each line is predicted to be unstable to helical buckling for the given $(L / a)$. Error bars show one standard deviation attributed to experimental conditions. Parameters from individual data points are given in the SM.

with a value equal to the average along the length of the segment,

$$
\tau_{\mathrm{av}}\left(s^{*}\right) L=12 \frac{\omega \eta}{E}\left(\frac{L}{a}\right)^{2}\left(2-\frac{s^{*}}{L}\right) .
$$

This average unitless torsion of the curve (i.e., radians of rotation along the total length) is maximum for short segments near the driven end (i.e., for small $s^{*}$ ), and it is quadratically dependent on the aspect ratio $L / a$. Continuing our dissection of the rod into $s^{*}$ segments and employing Eq. (2), a rod with zero tension will buckle when $\tau_{\mathrm{av}}\left(s^{*}\right) \geqslant \tau_{c}\left(s^{*}, 0\right)$, finally yielding

$$
\lim _{T \rightarrow 0} \omega_{c}=\frac{\pi}{4} \frac{E}{\eta}\left(\frac{a}{L}\right)^{2}
$$

when $s^{*}=L$. Though our model makes some ambitious assumptions, our numerical prefactor of $\pi / 4 \approx 0.785$ is comparable to the result of 0.563 from the standard model by Wolgemuth et al., which is based on numerical solutions of the linearized slender-body dynamics model $[15,16]$.

Next, we expand our model by modifying the tension component of Eq. (2) to include an axial fluid flow at a velocity $v$. This study assumes a laminar flow profile where experiments were conducted with Reynolds number $\ll 1$. The flow profile is assumed to be axial and laminar. The axial drag coefficient for a rod is $\zeta_{\|}=2 \pi \eta /[\ln (L / 2 a)+c]$, where the logarithmic denominator stems from Stokes' paradox, and accounts for the finite length of the rod [26]. The constant $c$ stems from additional terms in asymptotic expansion [25]. The axial tension on the rod is therefore $T=\zeta_{\|} v(L-s)$, and following the same steps that led to Eq. (4), we arrive at

$$
T_{\mathrm{av}}\left(s^{*}\right)=\frac{\pi \eta L v}{\ln (L / 2 a)-0.84}\left(2-\frac{s^{*}}{L}\right) .
$$

Inserting this average tension back into Eqs. (2) and (4), we arrive at the final governing inequality,

$$
12 \frac{\omega \eta}{E} \frac{L^{2}}{a^{2}}\left(2-\frac{s^{*}}{L}\right) \geqslant 3 \sqrt{\pi^{2} \frac{L^{2}}{s^{* 2}}+4 \alpha \frac{v \eta}{E a} \frac{L^{3}}{a^{3}} \frac{2-s^{*} / L}{\ln \left(\frac{L}{2 a}\right)+\beta}},
$$

where the left-hand side represents the average torsion of the curve of a segment $s^{*}$ of a filament of total length $L$, and the right-hand side represents the critical torsion of the curve required for the segment to helically buckle. We set $\beta=$ -0.84 , which is derived from the $O(\ln (L / a))$ term, and which is within $<1 \%$ of the extrema values for our experimental range of $L / a=(200,600)$ [25]. The sole fitting parameter $\alpha$ accounts largely for our model's assumption that the axial fluid flow only exerts axial tension on the filament, whereas in reality it also applies an effective torque on the filament to reorient any perturbations back to the $\hat{z}$ axis orientation.

From Eq. (7) there are two unitless parameters that regulate this instability. First is the relative rotational velocity's contribution to torque, $(\omega \eta / E)(L / a)^{2}$, and second is the relative fluid velocity's contribution to tension, $(v \eta / E a)(L / a)^{3}$. Numerically solving for the critical rotational velocity $\omega_{c}$ in Eq. (7) yields the lines in Fig. 2, which capture the observed physical behavior across a wide parameter range.

There are two important limits to consider. First is the limit $(v \eta / E a)(L / a)^{3} \gg 1$, which allows us to ignore the first term under the square root in Eq. (7) and produces a scaling of $\omega_{c} \sim$ $\sqrt{v}$. Second, the zero fluid velocity limit, $v \rightarrow 0$, reproduces the result of Eq. (5) with a linear dependence $\omega_{c} \sim v$. Our model's prefactor of $\pi / 4 \sim 0.785$ from Eq. (5) is within a factor of 2 of the experimental results, which appear to lie in the range of 0.3-0.4 (see the $y$ intercept in Fig. 2). 


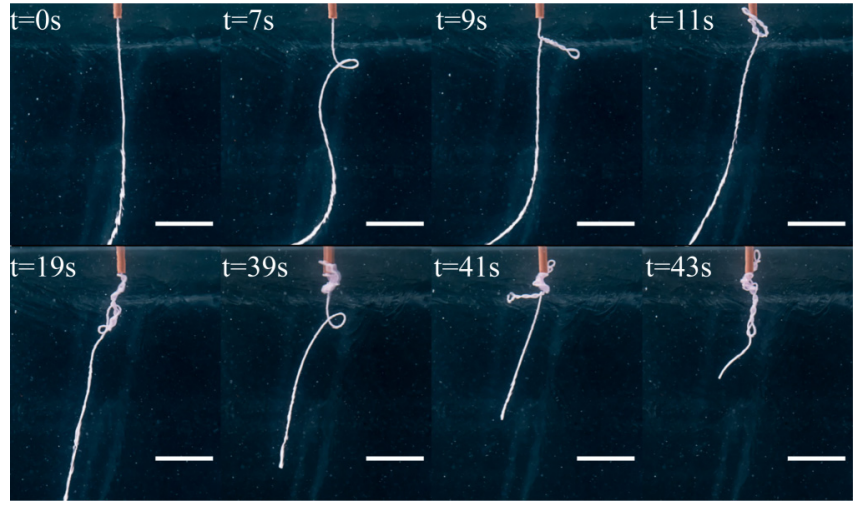

FIG. 3. Time series showing successive formation of three plectonemes in a filament with $L=10 \mathrm{~cm}, a=178 \mu \mathrm{m}$, and $\omega=$ $57 \mathrm{rad} / \mathrm{s}$. Plectonemes form at $\approx 7,19$, and $39 \mathrm{~s}$. Scale bars are all $1 \mathrm{~cm}$.

For further comparison to alternative existing numerical models, the experimental results yield an $\omega_{c}$ that is also below what was originally predicted by Wolgemuth et al. [15]; however, it is above the numerical results of Lim and Peskin's immersed-boundary fluid dynamics model [19]. Our own model can expect an overestimation of the prefactor because it was difficult to prepare the initial state of the filament to be perfectly straight, and some amount of curvature was always present (as seen in the $t=0$ images in Fig. 1). Wada and Netz have previously shown that the initial filament shape reduces $\omega_{c}$ by $25 \%$ for an initial radius of curvature of $L / 2 \pi$ [17], which is within the range of our experiments. Additionally, our approximation in Eq. (4) underestimates the average torsion by assuming it to be constant throughout the filament, while in reality the torsion is concentrated at the rotating (top) end. One would therefore expect an overestimation of this prefactor.

We made two additional experimental observations. First, the time required to reach the steady buckled state decreased with increasing $\omega$, as would be naively expected. Second, the location of buckling (and therefore plectoneme formation) was independent of $\omega$. This behavior is much like how the location of Euler buckling for a beam does not depend on the loading rate (for sufficiently slow rates), but rather only depends on if the critical load is achieved. Once the critical torsion had been exceeded in our system, the deflection occurred at a distance only dependent on the length scale that determined the bending rigidity $E I$; more specifically, the location of buckling depended only on the radius of the filament $a$.

Surprisingly, this feature holds true even when multiple plectonemes form within the same filament. Typically, the normal torquing of a rod at both ends leads to only a single plectoneme as the initial helical instability gives way to localized writhing $[23,27]$. This single plectoneme then grows in size, with further twisting only adding to its length. Alternatively, our unique setup of twisting via fluid drag allows for multiple plectonemes. An example of this behavior is shown in Fig. 3, which achieves a final total of three plectonemes. After the complete formation of the first plectoneme-whose final length is set by the available length of filament between the plectoneme's midpoint and the clamped top end-if there is sufficient length remaining to exceed the critical buckling torque, then another buckling event will occur at approximately the same distance from the rotating end. Note that after a plectoneme forms, it quickly wraps around the clamped end of the filament, effectively decreasing the total free length below available to make the next plectoneme. This phenomenon is an example where multiple plectonemes can be formed in a filament without directly manipulating nodal points between plectonemes, or allowing for entropic effects in the thermodynamic limit (such as for DNA) [28].

By trading twisting stress for bending stress, a single plectoneme effectively acts as a Hookean storage container for fiber length, much as the coil spring and spool of a tape measure. This behavior therefore transforms traditionally nonelastic fibers into elastic ones. Furthermore, the effective strain capacity is no longer constrained by the yield strength of the material itself but by the amount of length absorbed by the plectoneme. This remarkable behavior means that plectoneme-containing strands could be used to construct superelastic fibers and fabrics, opening another class of fiberbased artificial muscles used for work or actuation in soft materials [29]. Previously, only single-plectoneme fibers could be formed, which ensured unwieldy long plectonemes that would themselves behave as unruly strings, getting knotted, twisted, and tangled, and therefore preventing them from behaving as an ideal spring. However, by using our technique to divide plectonemes into many units within a fiber, the aforementioned problem is averted. Therefore, we envision multiplectoneme fibers to have many potential applications including soft robotics and smart textiles for advanced superelastic materials.

The authors would like to thank the Draper staff for their support on experiment development and analysis: Ernest Kim, David Adler, Peter H. Lewis, Vanessa Sanchez, Terrell Williams, Tim Cook, Jordan De Guzman, Robert Hodgson, Peter Yung, Seth Van Liew, and Amy Duwel. This work was sponsored by the Air Force Research Laboratory (AFRL) and the Defense Advanced Research Projects Agency (DARPA).
[1] C. H. Wiggins and R. E. Goldstein, Phys. Rev. Lett. 80, 3879 (1998).

[2] J. Elgeti, R. G. Winkler, and G. Gompper, Rep. Prog. Phys. 78, 056601 (2015).

[3] S. P. Timoshenko and J. M. Gere, Theory of Elastic Stability, Dover Civil and Mechanical Engineering (Dover, New York, 2012).
[4] B. Audoly and Y. Pomeau, Elasticity and Geometry: From Hair Curls to the Non-linear Response of Shells (Oxford University Press, Oxford, U.K., 2010).

[5] N. Leijnse, L. B. Oddershede, and P. M. Bendix, Proc. Natl. Acad. Sci. USA 112, 136 (2014).

[6] K. Son, J. S. Guasto, and R. Stocker, Nat. Phys. 9, 494 (2013). 
[7] J. L. Silverberg, R. D. Noar, M. S. Packer, M. J. Harrison, C. L. Henley, I. Cohen, and S. J. Gerbode, Proc. Natl. Acad. Sci. USA 109, 16794 (2012).

[8] M. Hajianmaleki and J. S. Daily, J. Pet. Sci. Eng. 116, 136 (2014).

[9] J.-S. Chen and H.-C. Li, J. Appl. Mech. 78, 41009 (2011).

[10] A. Aydin, L. Sun, X. Gong, K. Russell, D. Carter, and R. Gordon, ACS Appl. Polym. Mater. 1, 1717 (2019).

[11] K. J. Russell et al. (unpublished).

[12] F. P. Gosselin, P. Neetzow, and M. Paak, Phys. Rev. E 90, 052718 (2014).

[13] M. K. Jawed, N. K. Khouri, F. Da, E. Grinspun, and P. M. Reis, Phys. Rev. Lett. 115, 168101 (2015).

[14] See Supplemental Material at http://link.aps.org/supplemental/ 10.1103/PhysRevResearch.1.032020 for experiment details and data summary.

[15] C. W. Wolgemuth, T. R. Powers, and R. E. Goldstein, Phys. Rev. Lett. 84, 1623 (2000).

[16] T. R. Powers, Rev. Mod. Phys. 82, 1607 (2010).

[17] H. Wada and R. R. Netz, Europhys. Lett. 75, 645 (2006).

[18] H. Wada, Phys. Rev. E 84, 042901 (2011).
[19] S. Lim and C. S. Peskin, SIAM J. Sci. Comput. 25, 2066 (2004).

[20] S. D. Olson, S. Lim, and R. Cortez, J. Comput. Phys. 238, 169 (2013).

[21] T. C. Boles, J. H. White, and N. R. Cozzarelli, J. Mol. Biol. 213, 931 (1990).

[22] S. Forth, C. Deufel, M. Y. Sheinin, B. Daniels, J. P. Sethna, and M. D. Wang, Phys. Rev. Lett. 100, 148301 (2008).

[23] J. M. Thompson and A. R. Cliampneys, Proc. R. Soc. London, Ser. A 452, 117 (1996).

[24] A. Lazarus, J. T. Miller, M. M. Metlitz, and P. M. Reis, Soft Matter 9, 8274 (2013).

[25] J. B. Keller and S. I. Rubinow, J. Fluid Mech. 75, 705 (1976).

[26] H. Lamb, Hydrodynamics, Dover Books on Physics (Dover, New York, 1945).

[27] G. H. M. Van Der Heijden and J. M. T. Thompson, Nonlinear Dyn. 21, 71 (2000).

[28] M. Emanuel, G. Lanzani, and H. Schiessel, Phys. Rev. E 88, 022706 (2013)

[29] C. S. Haines, N. Li, G. M. Spinks, A. E. Aliev, J. Di, and R. H. Baughman, Proc. Natl. Acad. Sci. USA 113, 11709 (2016). 